\title{
The Secondary Development Based on MapX-energency Evacuation System Design and Implementation
}

\author{
Ying Su \\ School of Traffic and Transportation, Beijing Jiaotong University, Beijing 100044, China \\ suying317@163.com
}

\begin{abstract}
Since twenty-first century, earthquakes, floods and other disasters occurred frequently in the world. These disasters caused a huge loss of life and property. How to evacuate the personnel to safety area quickly and orderly has become particularly important. In order to realize the rapid and safe evacuation before the disaster, this paper uses the Dijkstra algorithm, based on the objectoriented programming idea, using the combination of Visual Basic and MapX development model to design the software. The results are helpful to improve the emergency command ability of the government traffic management department, and help the decision-makers to make scientific and reasonable decision in the construction of evacuation system and the process of evacuation.
\end{abstract}

Keywords: Emergency evacuation system; MapX; Shortest paths; Topological structure.

\section{Introduction}

With the acceleration of the urbanization process, the scale of urban population is gradually expanding. The potential safety hazards faced by urban development have become increasingly prominent. Earthquake, fire, flood, gas leakage and other emergency events are always threatening the safety of people's lives and property. Due to the high density of urban population and high concentration of wealth, any improper disposal of emergencies will cause significant casualties and property losses. Therefore, how to reduce the damage caused by urban emergencies and maintain the harmonious and stable development environment of cities is an important issue for governments of all countries.

At present, the overseas research on emergency evacuation mainly includes mathematical analysis method and computer simulation method in theory, and it can be divided into three types from the scale of evacuation model: macro model, medium model and micro model. It is a common method for emergency evacuation research to transform the large-scale emergency evacuation problem into the optimization problem of road network. Its deficiency is that the mathematical analysis method can only use static network flow, which cannot reflect the real-time condition of traffic, and the flexibility is poor. The breakthrough in emergency evacuation research is the application of computer simulation technology in this field. Compared with foreign countries, the research on personnel evacuation started relatively late in China, but developed quickly. Various cities issued emergency contingency plans successively, which made specific arrangements for personnel evacuation under emergencies, and also accelerated the construction of urban air-defense evacuation system.

\section{System Design}

The development of geographic information system software in this paper is taking Caofeidian region as the research area. Based on the object-oriented programming idea, the design is completed by using the development mode combining VB and MapX. As the circuit optimization software, the optimal path problem is equivalent to finding the shortest path problem in the network graph. This design adopts the Dijkstra algorithm, which is generally recognized at home and abroad.

\subsection{Software Development Tools}

This design adopts the development mode of combination of VB and MapX. VB is a visual, objectoriented, and event-driven structured high-level programming language, and is a simple and efficient tool for developing application software. MapX is the ActiveX control product of MapInfo which can 
provide users with powerful map analysis function. With MapX, users can simply and quickly embed mapping function in their own application, enhance the spatial analysis ability of the application, and realize the value-added of the application. In the initial design stage of the program, MapX control is first added to the form, then its properties are modified, and the corresponding methods and events are filled with code blocks, so that the geographic information data can be displayed in the form, further enriching the functions of the geographic information system.

\subsection{Main Work}

In the design process, the spatial geographic information data of the study area was collected first, and the road traffic network layer, road node layer and refuge layer were mapped with the Caofeidian area map as the base map, which were integrated into. The GST file was imported into VB for programming. Software functions include: map operation, path planning, personnel distribution, start and end point settings.

\section{Software Functional Module Design}

The main function module of the software is the shortest path algorithm. In this paper, Dijkstra algorithm is applied, and the module design of topological relation establishment, starting and ending setting and path track display is carried out.

\subsection{Research on Shortest Path Algorithm}

In network analysis, the analysis of shortest path problem is the most basic and the most critical. Nowadays, the methods to solve the shortest path analysis problems have been relatively mature, such as Dijkstra algorithm and Floyd algorithm, among which Dijkstra algorithm is the most classic and commonly used shortest path algorithm and is often used as the theoretical basis for solving the shortest path problem. The process of finding the shortest path by Dijkstra algorithm is the process of finding the adjacency point for each point. When each point has an adjacency point, and the route connected by this adjacency point is the shortest route, the search is completed.

\subsection{Establishment of Topological Relations}

To obtain the shortest path between any two points on the electronic map, the key is to build the corresponding topological structure map on the electronic map. The topology diagram consists of a number of points and the arcs (edges) between them, and the lengths of these arcs (edges) are known. On the basis of the topology diagram, the Dijkstra algorithm can be applied to calculate the shortest path.

In order to create the topological relation of point line, it is required that the intersecting arc in the spatial data must be broken at the intersection point, and then create the topological relation of point line by point. MapInfo cannot realize automatic disconnection of intersecting arc at the intersection point. Therefore, when making TAB data, attention should be paid to avoid intersections between arc and arc. When the arc segment intersects the arc segment, it can be manually disconnected at the junction. When establishing the point-line topological relation, loop all arcs, extract the head and tail node of each arc, and output the ID of arc, the ID of two endpoints of arc, and the length of arc in the document, as the data source for redefining OD matrix.

\subsection{Start and End Point Settings}

When looking for the shortest path, the starting point and end point may be on the road network or outside the road network: when both the starting point and the ending point are on the road network, Dijkstra algorithm can be directly used to calculate the shortest path from the beginning to the end point. When the starting point or end point is not in the road network, it is necessary to find the road node closest to the starting point or end point, and use this node as the starting point or end point to calculate the shortest path with Dijkstra algorithm. 


\subsection{Display Path Trajectories}

First, create a "distance shortest path" layer and add the line element to the layer. Before adding the layer, set its color and line width.

The part between start point and end point on the line element can be considered as a polyline formed by several short lines. We first create the ordered set $S$ of point graph elements that constitute graph elements between two points. Then we add the endpoint into S, find the Path(endpoint) of the precursor node of end point from the back forward and add it into $S$, and then loop it in turn, find the precursor node and add it to $\mathrm{S}$ until it is added to the starting point and end the loop. We take two consecutive points from front to back in S, find the public section at two points, copy the section to the new line element and set the colour and width of the line element. At last, add the line element to the created layer and display, and circle all points in S, and then end.

\section{Realization of Software Functions}

\subsection{Generation of Spatial Data}

The spatial geographic information data were collected by looking at the map of Caofeidian Industrial Zone. In Mapinfo, the grid image is opened in the form of opening table. After registration, five layers are drawn in turn: road layer, road node layer, shelter layer, gas station layer, gas station influence area layer. Run the GeoSet Manager software, open Layer Control, add the five layers drawn, save as the GeoSet file. As shown in figure 1.

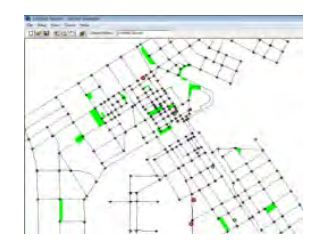

Fig. 1 GeoSet file

\subsection{Realization of Map Function}

\subsubsection{The Map Operation}

Start Visual Basic 6.0 software development tools for programming.

The map operation functions designed by this software include the following four. Zoom in: click on any position on the map, and the map will be displayed with the centre of the point at one time. Zoom out: when clicking on any position on the map, the map will be displayed with the point as the centre, and the scale will be doubled. Move: move the cursor to a certain position and drag the screen, and the map will move toward the drag direction. Layer control: it is the control of adding, deleting, moving and layer properties of the layer.

\subsubsection{Path Planning}

The software uses Dijkstra algorithm to calculate the shortest path. In order to complete this function, a total of 7 command buttons are set:

"Gas station fire area". You can click the button to display the fire area, and then click to restore the original oil site layer.

"No passing". If the fire range of the gas station is displayed, the program will look for the section within that range and add it to the section assembly, marking it out. At the same time, by looking for the number of nodes of each section in the set, if the number of nodes is equal to 2, the corresponding values of these two points in the OD matrix are set to 0 , that is, the length of the section is 0 and the passage is forbidden, and then the path optimization is carried out.

"Cancel". Using the ClearSelection method, unselect all the primitives in the fts_Selection collection, that is, the section is restored.

"The beginning". Select a point in a refuge, assign the centre point coordinates of the refuge location pixel to the point pixel, set the style of the point pixel and add it to the starting point. When 
adding the next starting point, if you select the same one, the MsgBox will pop up saying, "this location has been set as the starting point, please select another location". After adding the starting point correctly each time, the pop-up dialog box enters the number of people to be evacuated.

"The end". A point is selected at a place of refuge, the centre point coordinates of the place of refuge pixel are assigned to the point pixel, and the style of the point pixel is set and added to the end point. When adding the next endpoint, if you select the same one, the MsgBox will pop up saying "this location has been set as the endpoint, please select another location". Each time an endpoint is added, the refuge "quantity" and "mark" are set as its key fields and displayed.

"Start and end connections". The start and end match tool is used if the start and end points have been set, and there is at least one pair, otherwise the MsgBox prompt "please set the start and end points first" will pop up. When the button is used, the first point clicked by the mouse in a certain range of the refuge area around the starting mark is the starting point of the connecting line. Hold down the mouse, and drag to a certain range of the refuge area around the endpoint mark to loosen the finish line. A dialog box will pop up to enter the number of people assigned to that location.

"Path optimization". The button is only available when the starting endpoint is selected. With this button, each pair of starting endpoints performs the process of finding the nearest starting and ending nodes and calling the path calculation module (Dijkstra algorithm) and the path query and display module to complete the path optimization function. The software operation interface in the case of no road closure and road closure is shown in figure 2 and figure 3.

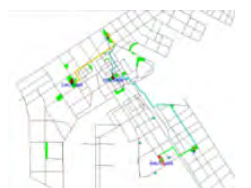

Fig. 2 Software running interface (without road closed)

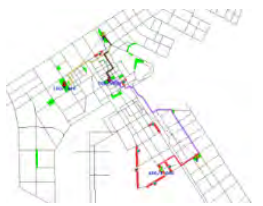

Fig. 3 Software running interface (with road closed)

\section{Summary}

Evacuation is an important measure to reduce the loss of life and property and maintain the public safety of the city. In this paper, MapX based centralized evacuation route optimization software is studied, and optimization innovation is carried out in terms of topological structure establishment, starting and ending point setting, and shortest path algorithm. The obtained results are of great significance for improving urban emergency response ability, enhancing government service ability and public trust. However, there are still some deficiencies in this study: the software does not design the function of cancelling the selection of starting point and end point, and will carry out program perfection after further study.

\section{References}

[1]. Xiaopeng Li, Bin Yu, Yamin Li. Shortest Path Search Algorithm Based on MapX. Computer Engineering and Design. Vol. 30 (2009) No. 22, p. 5225-5228.

[2]. Xuri Yin. Analysis and Applications of Shortest Path Based on MapX. Computer Knowledge and Technology. Vol. 5 (2009) No. 2, p. 477-479.

[3]. Jie Li. Visual Basic Programming Tutorial. Tsinghua University Press, 2011, p. 1-73. 
[4]. Yunqi Luo, Kun Zeng, Yi Luo. Construction of Digital Geographic Information System and Advanced Application of MapInfo. Tsinghua University Press, 2003, p. 341-418.

[5]. Anning Tang, XiuWan Chen, Caicong Wu. Best Path Analysis Based on MapX. Geography and Geo-Information Science. Vol. 26 (2010) No. 1, p. 29-33. 